\title{
HYDRODYNAMIC STATES OF ELECTRON PLASMA OF SEMICONDUCTORS IN THE GENERALIZED CHAPMAN-ENSKOG METHOD
}

\author{
S.A. Sokolovsky ${ }^{1}$, A.I. Sokolovsky², O.A. Hrinishyn ${ }^{2}$ \\ ${ }^{1}$ Prydniprovska State Academy of Civil Engineering and Architecture, Dnipro, Ukraine \\ ${ }^{2}$ Oles Honchar Dnipro National University, Dnipro, Ukraine \\ e-mail: alexander.i.sokolovsky@gmail.com
}

\begin{abstract}
The hydrodynamic states of polar semiconductors are investigated. Such states are described by the temperature, mass velocity, and density of the number of particles of the electron subsystem, which is considered as rarefied one. The phonon subsystem of a semiconductor is considered to be equilibrium. The electron-phonon interaction in the system is described by the Fröhlich Hamiltonian. The theory is based on Bogolyubov's linear kinetic equation for the electron distribution function in the presence of a weak electric field. The reduced description of the system is investigated by the generalized Chapman-Enskog method, which leads to a nonlinear equation for the electron distribution function in the reduced description. The zone structure of the system is neglected, focusing on the study of fundamental issues of relaxation of temperature and velocity. Gradients of hydrodynamic parameters are considered small; the same small parameters determine the smallness of the electric field. In the basic order of the perturbation theory, the distribution function of the system satisfies a nonlinear equation. The method of spectral theory of the collision integral operator shows that the study of the basic approximation is reduced to an analysis of a first-order quasilinear equation, which, in particular, has a solution that we found earlier.

Keywords: semiconductor, electron-phonon interaction, Bogolyubov kinetic equation, temperature and velocity relaxation, hydrodynamics, local equilibrium, collision integral operator, spectral theory, functional hypothesis.
\end{abstract}

Received 21.07.2020; Received in revised form 22.08.2020; Accepted 01.09.2020

\section{Introduction}

This work is devoted to the study of the influence of relaxation processes on hydrodynamic processes, which is an actual modern problem [1]. The problem is discussed in the framework of the theory of hydrodynamic states of the electron subsystem of polar semiconductors. It is assumed that the electron subsystem forms a rarefied gas, and the phonon subsystem is at rest and in equilibrium with temperature $T_{0}$. The paper investigates the fundamental issues of semiconductor kinetics and therefore neglects the crystal (band) structure of the system. In equilibrium, the state of such a system is spatially homogeneous and the electron energy is considered equal to $\varepsilon_{p}=p^{2} / 2 m$. Under such assumptions, Bogolyubov derived a linear kinetic equation for the electron-phonon system [2], which is the basis of this work. The same simplification is used in the monograph [3], which examines the theory of polarons.

We consider the hydrodynamic states of the electronic subsystem of a semiconductor, which are described by the energy density $\varepsilon(x, t)$, the momentum density $\pi_{l}(x, t)$, and the number of electrons $n(x, t)$ (or the density of their mass $\sigma(x, t)=m n(x, t))$. The velocity of the electron subsystem $u_{l}(x, t)$ and its temperature $T(x, t)$ are defined by formulas

$$
\pi_{l}(x, t)=\sigma(x, t) u_{l}(x, t), \quad \varepsilon(x, t)=\frac{3}{2} n(x, t) T(x, t)+\frac{1}{2} m n(x, t) u^{2}(x, t) .
$$

The first formula is not objectionable and is the velocity of the center of mass of the liquid element, as seen from the formula $\pi_{l}(x, t) d^{3} x=\sigma(x, t) d^{3} x u_{l}(x, t)$. The second formula is a consequence of the assumption of the local equilibrium, according to which the electron 
distribution function in main approximation $\mathrm{f}_{p}^{(0)}$ (in the spatially homogeneous state) coincides with the Maxwell distribution $w_{p-m u}(T)$, where

$$
w_{p}(T)=\frac{n}{(2 \pi m T)^{3 / 2}} e^{-\varepsilon_{p} / T} .
$$

This assumption cannot be true because the function $w_{p-m u}(T)$ is not a solution of the kinetic equation we use, even in the absence of an external electric field. The equilibrium solution at this condition is the Maxwell distribution without velocity and with phonon temperature $w_{p}\left(T_{0}\right) \equiv w_{p}$. Therefore, the assumption of local equilibrium can be approximately valid only when $w_{p-m u}(T) \approx w_{p}\left(T_{0}\right)$, i.e. at small values of $T-T_{0}$ and $u_{l}$ (see this in [4]). This means that the definition of the temperature of the electron subsystem of the semiconductor (2) is somewhat conditional. At the same time, temperature is a measure of the energy density of electrons in the accompanying reference. The simple formula (1) further also takes into account that the electron subsystem is an ideal gas. In this work, we study the hydrodynamic processes in semiconductors taking into account the relaxation $T \rightarrow T_{0}, u_{l} \rightarrow 0$, which, as will be shown below, is observed, despite a certain conditionality of the temperature definition (1). These processes are somewhat complicated in the presence of an external homogeneous constant electric field $E_{l}$, which will be considered small.

Note that in the framework of the concept of the local equilibrium, the relaxation of plasma temperatures was first studied by Landau [5]. A similar study of the relaxation of the velocities of the plasma components was performed in [6].

A number of our works were aimed developing approaches to the investigation of the hydrodynamic processes in the presence of relaxation without the assumption of the local equilibrium in the system. In [4] we summarized our idea of studying relaxation processes near their completion with going beyond the linearization of the basic equations of the theory. The next step was to develop and apply the spectral theory of the collision integral operator. In this way, the main approximation for the electron distribution function of the plasma case was found in the generalized Lorentz model [7-9]. It turned out that these ideas can also be applied to the study of polar semiconductors in the presence of a weak electric field, which is why our work is devoted.

The paper is constructed as it follows. Section 2 discusses the Bogolyubov kinetic equation and the spectral properties of the collision integral operator. Section 3 considers the basic equations of the Bogolyubov method of the reduced description for construction of the electron subsystem hydrodynamics that is a generalization of the Chapman-Enskog method. Section 4 discusses the theory of perturbations for the construction of hydrodynamics and derives the equations of hydrodynamics of an ideal liquid.

\section{Kinetic equation of the theory}

The work is based on a simplified model of a polar semiconductor as a system consisting of a nonequilibrium dilute subsystem of electrons and an equilibrium subsystem of non-interacting phonons with temperature $T_{0}$. The crystal (band) structure of the system is neglected, as it is a question of fundamental issues of taking into account in the theory of the transfer phenomena of relaxation processes of temperature and macroscopic velocities of subsystems equalization. The electron-phonon interaction is 
taken into account in the Fröhlich model. The kinetic equation for the distribution function of the model $\mathrm{f}_{p}(x, t)$

$$
\begin{gathered}
\partial_{t} \mathrm{f}_{p}(x, t)=-\frac{p_{l}}{m} \frac{\partial \mathrm{f}_{p}(x, t)}{\partial x_{l}}-F_{n} \frac{\partial \mathrm{f}_{p}(x, t)}{\partial p_{n}}+I_{p}\left(\mathrm{f}_{p^{\prime}}(x, t)\right), \\
\left(F_{n}=-e E_{n}, \quad \int d^{3} p \mathrm{f}_{p}(x, t)=n(x, t)\right)
\end{gathered}
$$

with the collision integral given by the formula

$$
\begin{gathered}
I_{p}\left(\mathrm{f}_{p^{\prime}}\right)=\frac{1}{(2 \pi)^{2} \hbar} \int d^{3} k g_{k}^{2}\left\{n_{k} \mathrm{f}_{p-k \hbar}-\left(1+n_{k}\right) \mathrm{f}_{p}\right\} \delta\left(\varepsilon_{p-k \hbar}+\hbar \omega_{k}-\varepsilon_{p}\right)+ \\
+\frac{1}{(2 \pi)^{2} \hbar} \int d^{3} k g_{k}^{2}\left\{\left(1+n_{k}\right) \mathrm{f}_{p+k \hbar}-n_{k} \mathrm{f}_{p}\right\} \delta\left(\varepsilon_{p}+\hbar \omega_{k}-\varepsilon_{p+k \hbar}\right)
\end{gathered}
$$

was derived from the first principles by the Bogolyubov method of eliminating boson variables [2] (see also our work [10]). Here, $n_{k}=\left(e^{\mathrm{h} \omega_{k} / T_{0}}-1\right)^{-1}$ is the Bose-Einstein distribution, $\omega_{k} \mathrm{~h}$ is phonon energy with a wave vector $k, \varepsilon_{p}=p^{2} / 2 m$ is electron energy with momentum $p_{l},-e$ is electron charge, $E_{n}$ denotes external constant homogeneous electric field, $n(x, t)$ is electron density. In the Fröhlich model suitable for describing polar crystals of semiconductors, the functions $\omega_{k}$ and $g_{k}$ are chosen as

$$
\omega_{k}=\omega, \quad g_{k}=2 \frac{\hbar \omega}{k}\left(\frac{\pi \alpha}{k_{0}}\right)^{1 / 2} \quad\left(k_{0} \equiv(m \omega / \hbar)^{1 / 2}\right)
$$

where $\alpha$ is the Fröhlich's dimensionless small constant, $k_{0}$ is a characteristic value of the wave number, $\omega$ is the characteristic frequency of the optical phonons (see, for example, [10]). The rareness of the electron subsystem leads to the linearity of the kinetic equation (3) and to the fact that the Maxwell distribution $w_{p} \equiv w_{p}\left(T_{0}\right)$ is its equilibrium solution $I_{p}\left(w_{p^{\prime}}\right)=0$ in the absence of the electric field.

The kinetic equation (3) is linear and therefore its solutions are convenient to discuss in the terms of the collision integral operator $\mathbf{K}$

$$
I_{p}\left(w_{p^{\prime}} a_{p^{\prime}}\right)=-w_{p} \mathbf{K} a_{p} .
$$

This operator is symmetric and positively defined one if the scalar product is introduced by the formula

$$
\left(a_{p}, b_{p}\right)=\left\langle a_{p} b_{p}\right\rangle \quad\left\langle a_{p}\right\rangle \equiv \int d^{3} p w_{p} a_{p}
$$

(see, for example, [11]; hereinafter $a_{p}, b_{p}$ are arbitrary functions). In the future, its eigenfunctions $g_{i p}$ and eigenvalues $\lambda_{i}$ will come in handy

$$
\mathbf{K} g_{i p}=\lambda_{i} g_{i p}, \quad \lambda_{i} \geq 0 .
$$

The eigenfunctions of the operator $\mathbf{K}$ form a complete orthonormal system and the solution of the kinetic equation can be sought in the form of the series

$$
\mathrm{f}_{p}=w_{p}\left(1+g_{p}\right), \quad g_{p}=\sum_{i} c_{i} g_{i p}, \quad\left(g_{i p}, g_{i^{\prime} p}\right)=b_{i} \delta_{i i^{\prime}}, \quad\left\langle g_{i p}\right\rangle=0
$$


$\left(b_{i}\right.$ are some positive normalization constants, the last formula follows from the normalization condition for $\mathrm{f}_{p}$ ).

\section{Construction of hydrodynamics of electron subsystem}

The equations of hydrodynamics are closed time equations for the densities of additive integrals of motion or their functions. In our case they are the densities of mass $\sigma(x, t)$, momentum $\pi_{l}(x, t)$ and energy $\varepsilon(x, t)$

$$
\sigma(x, t)=\int d^{3} p m \mathrm{f}_{p}(x, t), \quad \pi_{l}(x, t)=\int d^{3} p p_{l} \mathrm{f}_{p}(x, t), \quad \varepsilon(x, t)=\int d^{3} p \varepsilon_{p} \mathrm{f}_{p}(x, t)
$$

which describe nonequilibrium states of the system $(\sigma=m n)$. The corresponding time equations have the form of laws of change of these quantities

$$
\begin{gathered}
\frac{\partial \sigma(x, t)}{\partial t}=-\frac{\partial \pi_{n}(x, t)}{\partial x_{n}}, \quad \frac{\partial \pi_{l}(x, t)}{\partial t}=-\frac{\partial t_{n l}\left(\mathrm{f}_{p^{\prime}}(x, t)\right)}{\partial x_{n}}+R_{l}\left(\mathrm{f}_{p^{\prime}}(x, t)\right)+n(x, t) F_{l}, \\
\frac{\partial \varepsilon(x, t)}{\partial t}=-\frac{\partial q_{n}\left(\mathrm{f}_{p^{\prime}}(x, t)\right)}{\partial x_{n}}+R_{0}\left(\mathrm{f}_{p^{\prime}}(x, t)\right)+\frac{1}{m} \pi_{l}(x, t) F_{l} .
\end{gathered}
$$

Here $t_{n l}\left(\mathrm{f}_{p^{\prime}}\right), q_{n}\left(\mathrm{f}_{p^{\prime}}\right)$ are momentum and energy flux densities, $R_{l}\left(\mathrm{f}_{p^{\prime}}\right), R_{0}\left(\mathrm{f}_{p^{\prime}}\right)$ are sources that describe the relaxation processes associated with the interaction of subsystems. These quantities are given by the formulas

$$
\begin{array}{cc}
t_{n l}\left(\mathrm{f}_{p^{\prime}}\right)=\int d^{3} p p_{l} \frac{p_{n}}{m} \mathrm{f}_{p}, \quad q_{n}\left(\mathrm{f}_{p^{\prime}}\right)=\int d^{3} p \varepsilon_{p} \frac{p_{n}}{m} \mathrm{f}_{p} \\
R_{0}\left(\mathrm{f}_{p^{\prime}}\right)=\int d^{3} p \varepsilon_{p} I_{p}(\mathrm{f})=-\left\{\varepsilon_{p}, g_{p}\right\}, & R_{l}\left(\mathrm{f}_{p^{\prime}}\right)=\int d^{3} p p_{l} I_{p}(\mathrm{f})=-\left\{p_{l}, g_{p}\right\}
\end{array}
$$

(see (3), (5), (9)). These expressions contain useful in the future symmetric positively defined bilinear forms (integral brackets), which are determined for arbitrary functions by the formula

$$
\left\{a_{p}, b_{p}\right\}=\left\langle a_{p} \mathbf{K} b_{p}\right\rangle,
$$

(properties of this form are discussed in [11]).

As independent hydrodynamic variables, instead of the densities of additive integrals of motion $\sigma(x, t), \pi_{l}(x, t), \varepsilon(x, t)$ the mass velocity $u_{l}(x, t)$, mass density $\sigma(x, t)$ and temperature $T(x, t)$ of the electron subsystem can be chosen (they are defined by (1)). In the introduction, a certain limitation of such a definition of the temperature of the electron subsystem is discussed.

The construction of closed time equations for hydrodynamic variables $\xi_{\mu}(x): \varepsilon(x)$, $\pi_{l}(x), \sigma(x)$ is based in our work on the Bogolyubov functional hypothesis

$$
\mathrm{f}_{p}(x, t) \underset{t ? \tau_{\mathrm{fp}}}{\longrightarrow} \mathrm{f}_{p}(x, \xi(t))
$$

where $\tau_{\mathrm{fp}}$ is the free path time of electrons. The functional hypothesis is the basis of his method of the reduced description of nonequilibrium processes (see a review in [12]). It shows that at large times the electron distribution function has a structure $\mathrm{f}_{p}(x, \xi(t))$ where $\mathrm{f}_{p}(x, \xi)$ is a functional of functions $\xi_{\mu}(x)$. Wherein, it depends on time only 
through the hydrodynamic variables $\xi_{\mu}(x, t)$. This leads to closed time equations of the form

$$
\frac{\partial \xi_{\mu}(x, t)}{\partial t}=L_{\mu}\left(x, \mathrm{f}_{p^{\prime}}(\xi(t))\right)
$$

where the functional $L_{\mu}(x, \mathrm{f})$ is given by formulas (10) and (11). The kinetic equation (3) taking into account the functional hypothesis (13) and formula (14) gives the equation for the functional $\mathrm{f}_{p}(x, \xi)$

$$
\sum_{\mu} \int d^{3} x^{\prime} \frac{\delta \mathrm{f}_{p}(x, \xi)}{\delta \xi_{\mu}\left(x^{\prime}\right)} L_{\mu}\left(x^{\prime}, \mathrm{f}_{p^{\prime}}(\xi)\right)=-\frac{p_{n}}{m} \frac{\partial \mathrm{f}_{p}(x, \xi)}{\partial x_{n}}-F_{l} \frac{\partial \mathrm{f}_{p}(x, \xi)}{\partial p_{l}}+I_{p}\left(x, \mathrm{f}_{p^{\prime}}(\xi)\right)
$$

which can be called the kinetic equation in the reduced description. Definition (9) of parameters $\xi_{\mu}(x)$ that describe the state of the system gives additional conditions to equation (15)

$$
\int d^{3} p \mathrm{f}_{p}(x, \xi) \xi_{\mu p}=\xi_{\mu}(x) \quad \xi_{\mu p}: \varepsilon_{p}, p_{l}, m .
$$

Note that the additional conditions (16) together with equation (15) give expressions for the functionals $L_{\mu}(x, \mathrm{f})$. Therefore, when solving equation (15), we can combine some expressions for $L_{\mu}(x, \mathrm{f})$ with additional conditions $(16)$.

\section{Hydrodynamic perturbation theory: ideal liquid approximation}

When solving equation (15) with additional conditions (16), the right-hand side of the hydrodynamic equations $L_{\mu}\left(x, \mathrm{f}_{p^{\prime}}\right)$ and flux densities $q_{n}\left(x, \mathrm{f}_{p^{\prime}}(\xi)\right), t_{n l}\left(x, \mathrm{f}_{p^{\prime}}(\xi)\right)$ are sought in perturbation theory. The small parameter of the theory is introduced by estimating the gradients of the parameters $\xi_{\mu}(x)$ and the electric field $E_{l}$ as

$$
\frac{\partial^{s} \xi_{\mu}(x)}{\partial x_{l_{1}} \ldots \partial x_{l_{s}}} \sim g^{s}, \quad E_{l} \sim g \quad(g<<1)
$$

and the above-mentioned quantities are calculated in the form of a series in its powers

$$
\begin{array}{cr}
\mathrm{f}_{p}(x, \xi)=\mathrm{f}_{p}^{(0)}+\mathrm{f}_{p}^{(1)}+O\left(g^{2}\right), & L_{\mu}(x, \mathrm{f}(\xi))=L_{\mu}^{(0)}+L_{\mu}^{(1)}+L_{\mu}^{(2)}+O\left(g^{3}\right), \\
q_{n}(x, \mathrm{f}(\xi))=q_{n}^{(0)}+q_{n}^{(1)}+O\left(g^{2}\right), & t_{n l}(x, \mathrm{f}(\xi))=t_{n l}^{(0)}+t_{n l}^{(1)}+O\left(g^{2}\right)
\end{array}
$$

(further more descriptive notation will be used $\left.L_{\mu}^{(s)} \equiv\left(\partial \xi_{\mu} / \partial t\right)^{(s)}\right)$. Here the small parameter is estimated by the formula $g=l_{\mathrm{fp}} / L$, where $l_{\mathrm{fp}}$ is free path length and $L$ is the characteristic length of spatial inhomogeneities of parameters $\xi_{\mu}(x)$ in the system (see, for example, [12]).

In the main approximation in the parameter $g$, the function $\mathrm{f}_{p}^{(0)}$ satisfies the equation

$$
\frac{\partial \mathrm{f}_{p}^{(0)}}{\partial \pi_{l}} R_{l}\left(\mathrm{f}_{p^{\prime}}^{(0)}\right)+\frac{\partial \mathrm{f}_{p}^{(0)}}{\partial \varepsilon} R_{0}\left(\mathrm{f}_{p^{\prime}}^{(0)}\right)=I_{p}\left(\mathrm{f}_{p^{\prime}}^{(0)}\right)
$$

and additional conditions

$$
\int d^{3} p \mathrm{f}_{p}^{(0)} \varepsilon_{p}=\varepsilon, \quad \int d^{3} p \mathrm{f}_{p}^{(0)} p_{l}=\pi_{l}, \quad \int d^{3} p \mathrm{f}_{p}^{(0)} m=\sigma .
$$


In the terms of contribution $g_{p}^{(0)}$ to the function $g_{p}$ equations (19) and (20) with definition (8) take the form

$$
\begin{gathered}
\frac{\partial g_{p}^{(0)}}{\partial \pi_{l}}\left\{p_{l}, g_{p}^{(0)}\right\}+\frac{\partial g_{p}^{(0)}}{\partial \varepsilon}\left\{\varepsilon_{p}, g_{p}^{(0)}\right\}=\mathbf{K} g_{p}^{(0)} ; \\
\left\langle\varepsilon_{p} g_{p}^{(0)}\right\rangle=\varepsilon-\varepsilon_{0}, \quad\left\langle p_{l} g_{p}^{(0)}\right\rangle=\pi_{l} \quad\left(\varepsilon_{0} \equiv 3 n T / 2\right)
\end{gathered}
$$

The solutions of these equations, the first of which is nonlinear, can be sought in the form of the expansion (8) of the function $g_{p}^{(0)}$ in the operator $\mathbf{K}$ eigenfunctions $g_{i p}$. Further for simplifying the consideration with rotational invariance arguments its eigenfunctions are chosen, following [9], in the form of irreducible polynomials

$$
C(p) \equiv A_{p}, \quad C_{l}(p) \equiv B_{p} p_{l}, \quad C_{l m}(p) \equiv C_{p}\left(p_{l} p_{m}-\frac{1}{3} p^{2} \delta_{l m}\right), \ldots C_{l_{1} \ldots l_{s}}(p) \ldots
$$

wherein the general polynomial is symmetric with zero convolution on any pair of its indices. The scalar and vector eigenfunctions are of the most importance for our study.

They satisfy the equations

$$
\mathbf{K} A_{p}=\lambda_{T} A_{p}, \quad \mathbf{K} B_{p} p_{l}=\lambda_{u} B_{p} p_{l} \quad\left(\left\langle A_{p}\right\rangle=0\right)
$$

with additional conditions

$$
\left\langle A_{p} \varepsilon_{p}\right\rangle=3 n / 2, \quad\left\langle B_{p} \varepsilon_{p}\right\rangle=3 n / 2,
$$

which fix an arbitrary multiplier in expressions (23) for functions $A_{p}, B_{p}$. At the same time, formulas (23) determine the corresponding eigenvalues $\lambda_{T} \mathrm{i} \lambda_{u}$. Note that the quantities $A_{p}, B_{p}$ and $\lambda_{T}, \lambda_{u}$ do not depend on the electron density $n$.

Thus, we find the solution of equations (21) in the form of an expansion

$$
g_{p}^{(0)}=c_{T}^{(0)} A_{p}+c_{u_{l}}^{(0)} B_{p} p_{l}+\sum_{i \neq T, u_{l}} c_{i}^{(0)} g_{i p} .
$$

Note further that the formulas which are a consequence of considerations of rotational invariance and the definition of an irreducible tensor

$$
\left\langle\varepsilon_{p} g_{i p}\right\rangle=0, \quad\left\langle p_{l} g_{i p}\right\rangle=0 \quad\left(i \neq T, u_{l}\right)
$$

are valid. According to the last two formulas in (21) relations (26) show that

$$
c_{T}^{(0)}=\left(\varepsilon-\varepsilon_{0}\right) 2 / 3 n, \quad c_{u_{l}}^{(0)}=\pi_{l} / m n .
$$

With account for (26), (27) the brackets included in (21) take the form

$$
\begin{gathered}
R_{0}^{(0)} \equiv-\left\{\varepsilon_{p}, g_{p}^{(0)}\right\}=-c_{T}^{(0)} \lambda_{T} 3 n / 2=-\lambda_{T}\left(\varepsilon-\varepsilon_{0}\right) \\
R_{l}^{(0)} \equiv-\left\{p_{l}, g_{p}^{(0)}\right\}=-c_{u_{l}}^{(0)} \lambda_{u} m n=-\lambda_{u} \pi_{l},
\end{gathered}
$$

that is, the first equation (21) is linear one (these brackets in accordance with (11) give the sources in the equations of hydrodynamics). Now equation (21) for $g_{p}^{(0)}$ is reduced to such a system of equations for coefficients $c_{i}^{(0)}$

$$
\frac{\partial c_{i}^{(0)}}{\partial \varepsilon} \lambda_{T}\left(\varepsilon-\varepsilon_{0}\right)+\frac{\partial c_{i}^{(0)}}{\partial \pi_{l}} \lambda_{u} \pi_{l}=\lambda_{i} c_{i}^{(0)} .
$$


For $i=T, u_{l}$ this relation, taking into account (27), holds identically and for $i \neq T, u_{l}$ gives the equation for the functions $c_{i}^{(0)}$. Equation (29) has a zero solution, which gives such an exact solution of equation (21) for the distribution function of the main approximation $\mathrm{f}_{p}^{(0)}$ in the small parameter of the theory

$$
\mathrm{f}_{p}^{(0)}=w_{p}\left(1+g_{p}^{(0)}\right), \quad g_{p}^{(0)}=\frac{2}{3 n} A_{p}\left(\varepsilon-\varepsilon_{0}\right)+\frac{1}{m n} B_{p} p_{n} \pi_{n}
$$

that was first obtained in our work [8] (see also [9]).

Now you can calculate the energy and momentum fluxes in the main approximation by formulas (11) and (30)

$$
q_{l}^{(0)}=\int d^{3} p w_{p}\left(1+g_{p}^{(0)}\right) \varepsilon_{p} p_{l} / m, \quad t_{n l}^{(0)}=\int d^{3} p w_{p}\left(1+g_{p}^{(0)}\right) p_{n} p_{l} / m
$$

that gives

$$
q_{l}^{(0)}=\frac{2}{3 \sigma}\left\langle\varepsilon_{p}^{2} B_{p}\right\rangle \pi_{l}, \quad t_{n l}^{(0)}=\frac{2}{3} \varepsilon \delta_{n l} .
$$

The equations of hydrodynamics of the zero and first approximations according to (10), (11), and (28) take the form

$$
\begin{gathered}
\left(\frac{\partial \varepsilon}{\partial t}\right)^{(0)}=-\lambda_{T}\left(\varepsilon-\varepsilon_{0}\right), \quad\left(\frac{\partial \pi_{l}}{\partial t}\right)^{(0)}=-\lambda_{u} \pi_{l} ; \\
\left(\frac{\partial \varepsilon}{\partial t}\right)^{(1)}=-\frac{2}{3}\left\langle\varepsilon_{p}^{2} B_{p}\right\rangle \frac{\partial}{\partial x_{l}} \frac{\pi_{l}}{\sigma}+\frac{1}{m} \pi_{l} F_{l}-\left\{\varepsilon_{p}, g_{p}^{(1)}\right\}, \\
\left(\frac{\partial \pi_{l}}{\partial t}\right)^{(1)}=-\frac{2}{3} \frac{\partial \varepsilon}{\partial x_{l}}+\frac{1}{m} \sigma F_{l}-\left\{p_{l}, g_{p}^{(1)}\right\}, \quad \frac{\partial \sigma}{\partial t}=-\frac{\partial \pi_{l}}{\partial x_{l}}
\end{gathered}
$$

(the last time equation for the mass density is exact). The first two equations describe relaxation processes in semiconductors in a spatially homogeneous case and in the absence of an external electric field with characteristic relaxation times $\tau_{T} \equiv \lambda_{T}^{-1}$, $\tau_{u} \equiv \lambda_{u}^{-1}$. In contrast to standard hydrodynamics, (33) includes the contribution $g_{p}^{(1)}$ to the function $g_{p}$, which usually describes only dissipative processes in the system (they enter in the second-order hydrodynamic equations in the small parameter of the theory).

\section{Conclusions}

Basing on the Bogolyubov's kinetic equation, semiconductors are studied as systems of a dilute nonequilibrium subsystem of electrons and an equilibrium system of phonons in a homogeneous constant electric field. The state of the electron subsystem is considered as a hydrodynamic one and is described by the densities of its energy, momentum and mass or their functions. Hydrodynamic phenomena are studied in the presence of relaxation processes of equalization of macroscopic velocities and temperatures of subsystems. It is noted that the system is not in a state of the local equilibrium and, in particular, the local temperature of the electrons has a somewhat limited meaning. The paper uses the results of our studies of completely ionized plasma in the generalized Lorentz model [9]. The exact spatially homogeneous solution of the kinetic equation is found by the method of spectral theory of the collision integral operator $\mathbf{K}$ and replaces the inadequate Maxwell distribution with the temperature and 
velocity of the electron subsystem. The solution is formulated in the terms of its scalar and vector eigenfunctions, which can be found only approximately. The nonequilibrium state of the system is investigated on the basis of the Bogolyubov functional hypothesis by a method that generalizes the Chapman-Enskog method. It is assumed that the gradients of hydrodynamic variables and the electric field are small quantities of the same order. In the presence of relaxation in the system caused by the electron-phonon interaction, the equations of hydrodynamics have the form of the laws of change of its energy and momentum densities (conservation laws with sources). It is important to note that the sources in the equations of hydrodynamics in the ideal liquid approximation are given by the same contribution to the nonequilibrium electron distribution function, which one needs for investigating the standard dissipative processes.

\section{References}

1. Jou D. Extended irreversible thermodynamics / D. Jou, J. Casas-Vázques, G. Lebon. - N.Y.: Springer, 2010. -483 p.

2. Bogolyubov, N.N. Kinetic equations for the electron-phonon system / N.N. Bogolyubov. - Dubna: JINR, Preprint E17-11822, 1978. - 70 p.

3. Bogoylubov, N.N. Aspects of Polaron Theory. Equilibrium and Nonequilibrium Problems / N.N. Bogoylubov, N.N. Bogolyubov (Jr.). - Singapore: World Scientific, 2008. $-180 \mathrm{p}$.

4. Gorev, V.N. New method in the theory of relaxation processes for nonhomogenous media / V.N Gorev, S.A. Sokolovsky, A.I. Sokolovsky // Visnyk of Dnipropetrovsk University. Physics, Radioelectronics. - 2016. - Vol. 24. - P. 83 - 93.

5. Landau, L.D. Kineticheskoe uravnenie v sluchae kulonovskogo vzaimodejstviya / L.D. Landau // ZhETF. - 1936. - Vol. 7. - P. 203 - 209 (in Russian).

6. Alexandrov, A.F. Osnovy elekrodinamiki plazmy / A.F. Aleksandrov, L.S. Bogdankevich, A.A. Rukhadze // Moscow: Vysshaya shkola. - 1988. - 424 p. (in Russian).

7. Sokolovsky, S.A. Relaxation processes in completely ionized plasma in generalized Lorentz model / S.A. Sokolovsky, A.I. Sokolovsky, I.S. Kravchuk, O.A. Grinishin / Journal of Physics and Electronics. - 2018. - Vol. 26(2). - P. 17 - 28; doi.org / 10.15421/331818.

8. Sokolovsky, S. Mobility of electrons in plasma / S. Sokolovsky, A. Sokolovsky // 2019 IEEE 2nd Ukraine Conference on Electrical and Computer Engineering (UKRCON-2019) (July 2-6, 2019, Lviv, Ukraine) Proceedings, ISBN: 978-1-7281-3882-4. - P. 783 - 787.

9. Sokolovsky A.I. On relaxation processes in a completely ionized plasma / A.I. Sokolovsky, S.A. Sokolovsky, O.A. Hrinishyn // East Eur. J. Phys. - 2020. - Vol. 3. P. 19 - 30; doi.org / 10.26565/2312-4334-2020-3-03.

10. Sokolovsky, S.A. Toward polaron kinetics in the Bogolyubov reduced description method / S.A. Sokolovsky // Theoretical and Mathematical Physics. - 2011. - Vol. 168, No. 2. - P. 1150 - 1164.

11. Sokolovsky, S.A. On theory of nonlinear relaxation in polaron subsystem of polar semiconductors / S.A. Sokolovsky // Proceedings of XXII ${ }^{\text {nd }}$ International Seminar/Workshop on direct and inverse problems of electromagnetic and acoustic wave theory (DIPED) (September 25-28, 2017, Dnipro, Ukraine). - 2017. - P. 242 - 247.

12. Akhiezer, A.I. Methods of Statistical Physics / A.I. Akhiezer, S.V. Peletminskii. -Amsterdam: Elsevier. - 2013. - 446 p. 